\title{
Recursive Data Analysis in Large Scale Complex Systems
}

\author{
Esko K. Juuso \\ Control Engineering, Faculty of Technology, University of Oulu, Finland, esko. juuso@oulu.fi
}

\begin{abstract}
Advanced data analysis is needed in practical applications in large scale complex systems. Variable specific datadriven solutions provide consistent levels, which can be used in compact model structures. In changing operating conditions, the recursive analysis extends the applicability of these structures in building and tuning dynamic and case-based models for complex systems since the meanings change more frequently than the interactions. The methodology provides information about uncertainty, fluctuations and confidence in results. The scaling approach brings temporal analysis to all measurements and features: trend indices are calculated by comparing the averages in the long and short time windows, a weighted sum of the trend index and its derivative detects the trend episodes and severity of the trend is estimated by including also the variable level in the sum. The trend episodes and temporal adaptation of the scaling functions with time are used in the early detection of changes in the operating conditions. The levels are understood as fuzzy labels and the decision making is based on fuzzy calculus. The solution is highly compact: all variables, features and indices are transformed to the range $[-2,2]$ and represented in natural language which is important in integrating datadriven solutions with domain expertise.
\end{abstract}

Keywords: recursive data analysis, nonlinear scaling, temporal analysis, fuzzy set systems, large scale systems

\section{Introduction}

The steady-state simulation models can be relatively detailed nonlinear multiple input, multiple output (MIMO) models $\vec{y}=F(\vec{x})$, where the output vector $\vec{y}=$ $\left(y_{1}, y_{2}, \ldots, y_{n}\right)$ is calculated by a nonlinear function $F$ from the input vector $\vec{x}=\left(x_{1}, x_{2}, \ldots, x_{m}\right)$. More generally, the relationship could also be a table or a graph. Fuzzy set systems, artificial neural networks and neurofuzzy methods provide additional methodologies for the function $F(\vec{x})$.

Statistical modelling methodologies provide a wide variety of models based on linear regression. In the response surface methodology (RSM), the relationships are represented with multiple input, single output (MISO) models, which contain linear, quadratic and interactive terms (Box and Wilson, 1951). Application areas can be extended by arbitrary nonlinear models, e.g. semi-physical models, developed by using appropriate calculated variables as inputs (Ljung, 1999).
Principal component analysis (PCA) reduces the number of dimensions by using linear combinations of the original variables (Jolliffe, 2002). Partial least squares regression (PLS) uses potentially collinear variables (Gerlach et al., 1979). Nonparametric models for $y_{i}$ at each $\vec{x}$ can be constructed from data as a weighted average of the neighbouring values of $y_{i}$ (Wasserman, 2007)

Fuzzy set systems, which focus on the linguistic meanings of the variables, suit very well to qualitative descriptions of the process as they can be interpreted by using natural language, heuristics and common sense knowledge. Fuzzy logic emerged from approximate reasoning by maintaining clear connections with fuzzy rulebased systems and expert systems (Dubois et al., 1999). Fuzzy set theory first presented by Zadeh (Zadeh, 1965) form a conceptual framework for linguistically represented knowledge.

The extension principle is the basic generalisation of the arithmetic operations if the inductive mapping is a monotonously increasing function of the input. The interval arithmetic presented by Moore (Moore, 1966) is used together with the extension principle for evaluating fuzzy expressions (Buckley and Qu, 1990; Buckley and Hayashi, 1999; Buckley and Feuring, 2000). The fuzzy sets can be modified by intensifying or weakening modifiers (De Cock and Kerre, 2004).

Type-2 fuzzy models take into account uncertainty about the membership function (Mendel, 2007). Most systems based on interval type- 2 fuzzy sets are reduced to an interval-valued type-1 fuzzy set. Fuzzy set systems can also handle contradictory data (Krone and Kiendl, 1994; Krone and Schwane, 1996). Takagi-Sugeno (TS) fuzzy models (Takagi and Sugeno, 1985) combine fuzzy rules and local lineal models.

Linguistic equation (LE) approach combines datadriven methodologies with linguistic meanings. The LE approach originates from fuzzy set systems (Juuso and Leiviskä, 1992): rule sets are replaced with equations, and meanings of the variables are handled with scaling functions which have close connections to membership functions (Juuso, 1999). The nonlinear scaling technique is needed in constructing nonlinear models with linear equations (Juuso, 2004). Constraints handling (Juuso, 2009) and data-based analysis (Juuso and Lahdelma, 2010), improve possibilities to update the scaling functions recursively (Juuso, 2011a; Juuso and Lahdelma, 2011).

Combined fuzzy systems can include fuzzy arithemetics and inequalities (Juuso, 2014). Natural language in- 
terface is based on the scaling functions (Juuso, 2016). Temporal reasoning is a very valuable tool to diagnose and control slow processes: the LE based trend analysis introduced in (Juuso, 2011b) transforms the fuzzy rulebased solution (Cheung and Stephanopoulos, 1990) to an equation-based solution.

Smart adaptive systems (SAS) are aimed for developing successful applications in different fields. Three levels of adaptation have been identified (Anguita, 2001):

1. adaptation to a changing environment;

2. adaptation to a similar setting without explicitly being ported to it;

3. adaptation to a new or unknown application.

In the first level, a short-term memory is needed for incremental or on-line learning, a long-term memory for recognising context drifting. Successful past solutions and the idea of reasoning by analogy are used in the second level. The most challenging requirement is to adapt to new applications. In real applications, the constraint of starting from zero knowledge is modified to building new knowledge or, at least, improving the existing one. Adaptive fuzzy control proceeds through three stages: first scaling, then the shape of membership functions and finally rulebase. The LE approach has a similar preference sequence: scaling, shape of scaling functions and interaction equations.

This paper discusses the recursive data analysis based on the LE approach as a solution in the gradual refinement of large scale complex systems.

\section{Data analysis}

The nonlinearities of the process are handled by the nonlinear scaling of the variables. The parameters of the scaling functions are obtained by data analysis based on generalised norms and moments.

\subsection{Nonlinear scaling}

Scaling functions are monotonously increasing functions $x_{j}=f\left(X_{j}\right)$ where $x_{j}$ is the variable and $X_{j}$ the corresponding scaled variable. The function $f()$ consist of two second order polynomials, one for the negative values of $X_{j}$ and one for the positive values, respectively. The corresponding inverse functions $x_{j}=f^{-1}\left(X_{j}\right)$ based on square root functions are used for scaling to the range $[-2,2]$, denoted linguistification. In LE models, the results are scaled to the real values by using the function $f($ ).

The parameters of the functions are extracted from measurements by using generalised norms and moments. The support area is defined by the minimum and maximum values of the variable, i.e. the support area is $\left[\min \left(x_{j}\right), \max \left(x_{j}\right)\right]$ for each variable $j, j=1, \ldots, m$. The central tendency value, $c_{j}$, divides the support area into two parts, and the core area is defined by the central tendency values of the lower and the upper part, $\left(c_{l}\right)_{j}$ and $\left(c_{h}\right)_{j}$, correspondingly. This means that the core area of the variable $j$ defined by $\left[\left(c_{l}\right)_{j},\left(c_{h}\right)_{j}\right]$ is within the support area.

The generalised norm is defined by

$$
\left\|{ }^{\tau} M_{j}^{p}\right\|_{p}=\left(M_{j}^{p}\right)^{1 / p}=\left[\frac{1}{N} \sum_{i=1}^{N}\left(x_{j}\right)_{i}^{p}\right]^{1 / p}
$$

where the order of the moment $p \in R$ is non-zero, and $N$ is the number of data values obtained in each sample time $\tau$. The norm (1) calculated for variables $x_{j}, j=1, \ldots, n$, have the same dimensions as the corresponding variables. The norm $\left\|{ }^{\tau} M_{j}^{p}\right\|_{p}$ can be used as a central tendency value if all values $x_{j}>0$, i.e. $\left\|{ }^{\tau} M_{j}^{p}\right\|_{p} \in R$. (Lahdelma and Juuso, 2011). The norm can be extended to variables including negative values (Juuso, 2011a).

The orders, $p$, corresponding to the corner points are chosen by using the generalised skewness,

$$
\left(\gamma_{k}^{p}\right)_{j}=\frac{1}{N \sigma_{j}^{k}} \sum_{i=1}^{N}\left[\left(x_{j}\right)_{i}-\left\|^{\tau} M_{j}^{p}\right\|_{p}\right]^{k}
$$

The standard deviation $\sigma_{j}$ is the norm (1) with the order $p=2$. (Juuso and Lahdelma, 2010)

\subsection{Interactions}

The basic form of the linguistic equation (LE) model is a static mapping in the same way as fuzzy set systems and neural networks, and therefore dynamic models will include several inputs and outputs originating from a single variable (Juuso, 2004). External dynamic models provide the dynamic behaviour, and LE models are developed for a defined sampling interval in the same way as in various identification approaches discussed in (Ljung, 1999).

Adaptation of the nonlinear scaling is the key part in the data-based LE modelling (Figure 1). All variables can be analysed in parallel with the methodology described above and assessed with domain expertise. Interactions are analysed with linear modelling methodologies from the scaled data in the chosen time period. In large-scale systems, a huge number of alternatives need to be compared, e.g. in a paper machine application, 72 variables produced almost 15 million three to five variable combinations. Correlations and causalities based on domain expertise are needed to find feasible variable groups (Juuso and Ahola, 2008).

Dynamic LE models use the parametric model structures, ARX, ARMAX, NARX etc., but the nonlinear scaling reduces the number of input and output signals needed for the modelling of nonlinear systems. For the default LE model, all the degrees of the polynomials become very low:

$$
Y(t)+a_{1} Y(t-1)=b_{1} U\left(t-n_{k}\right)+e(t)
$$

for the scaled variables $Y$ and $U$. 


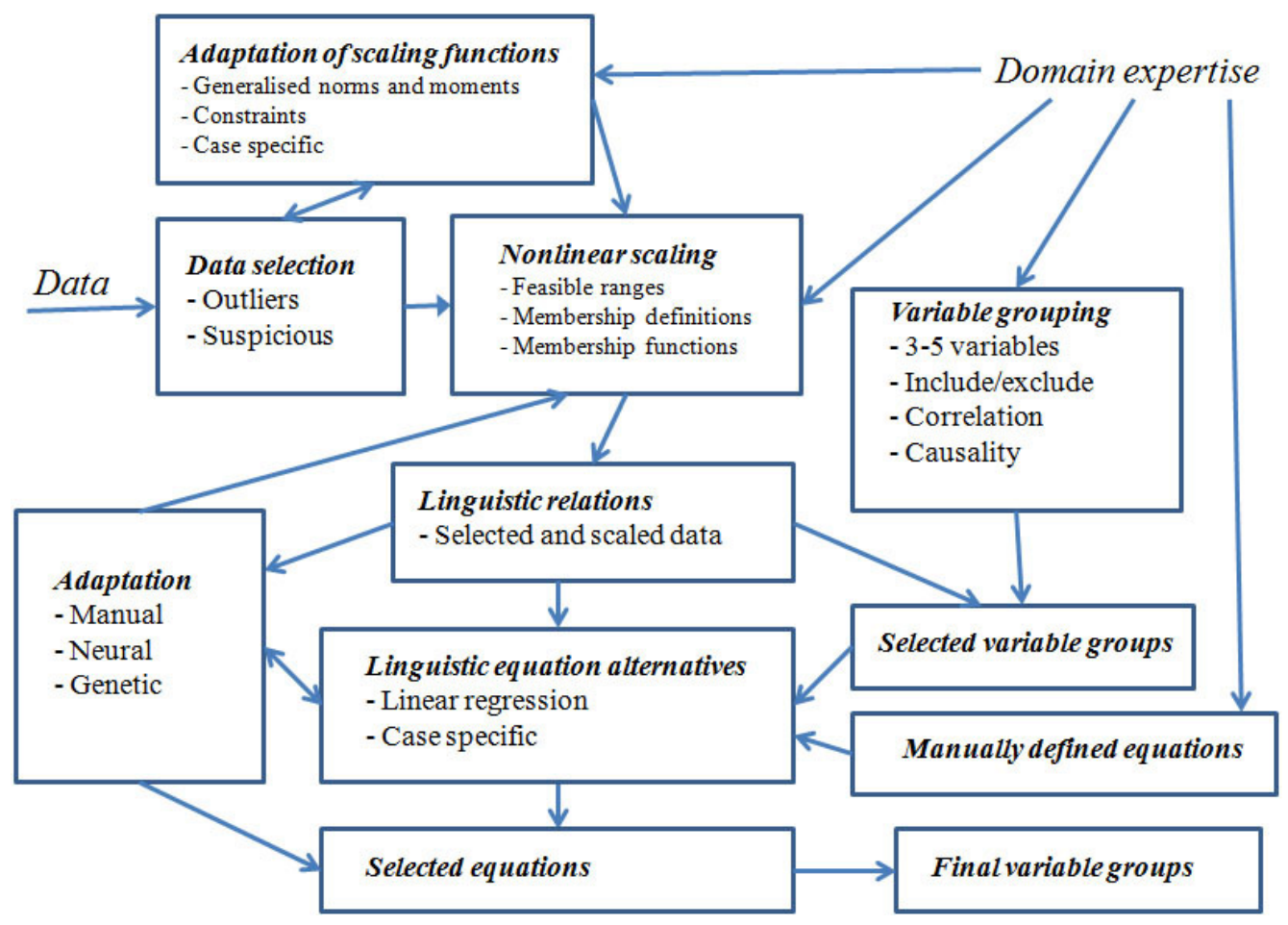

Figure 1. Data-based modelling with linguistic equations (Juuso, 2013).

\subsection{Uncertainty}

The norm values obtained from different time periods can have differences, i.e. the parameters of the scaling functions can be represented as fuzzy numbers. Thus the feasible range is defined a type- 2 trapezoidal membership function. A strong increase in uncertainty may demonstrate a change of operating conditions. The scaling functions monotonous and increasing if the ratios,

$$
\begin{aligned}
\alpha_{j}^{-} & =\frac{\left(c_{l}\right)_{j}-\min \left(x_{j}\right)}{c_{j}-\left(c_{l}\right)_{j}}, \\
\alpha_{j}^{+} & =\frac{\max \left(x_{j}\right)-\left(c_{h}\right)_{j}}{\left(c_{h}\right)_{j}-c_{j}},
\end{aligned}
$$

are both in the range $\left[\frac{1}{3}, 3\right]$, see (Juuso, 2009).

The coefficients of the second order polynomials can be represented by

$$
\begin{aligned}
& a_{j}^{-}=\frac{1}{2}\left(1-\alpha_{j}^{-}\right) \Delta c_{j}^{-}, \\
& b_{j}^{-}=\frac{1}{2}\left(3-\alpha_{j}^{-}\right) \Delta c_{j}^{-}, \\
& a_{j}^{+}=\frac{1}{2}\left(\alpha_{j}^{+}-1\right) \Delta c_{j}^{+}, \\
& b_{j}^{+}=\frac{1}{2}\left(3-\alpha_{j}^{+}\right) \Delta c_{j}^{+},
\end{aligned}
$$

where $\Delta c_{j}^{-}=c_{j}-\left(c_{l}\right)_{j}$ and $\Delta c_{j}^{+}=\left(c_{h}\right)_{j}-c_{j}$.

The ratios $\alpha_{j}^{-}$and $\alpha_{j}^{-}$are calculated with interval arithmetic. The constrant range $\left[\frac{1}{3}, 3\right]$ must be taken into account before calculating the coefficients (5). Also the extension principle is needed when calculating the scaled values as fuzzy numbers.

\subsection{Natural language}

All the scaled variables are in the same range $[-2,2]$ where integer numbers correspond labels, e.g. \{very low, low, normal, high, very high\}. Fuzzy numbers can be modified by fuzzy modifiers, which are used as intensifying adverbs (very, extremely) or weakening adverbs (more or less, roughly). The resulting terms,

$$
A_{1} \subseteq A_{2} \subseteq A_{3} \subseteq A_{4} \subseteq A_{5},
$$

correspond to the powers of the membership in the powering modifiers (Table 1). The vocabulary can also be chosen in a different way, e.g. highly, fairly, quite ( Juuso, 2012a). Only the sequence of the labels is important. Linguistic variables can be processed with the conjunction (and), disjunction (or) and negation (not). More examples can be found in (De Cock and Kerre, 2004).

For a time period, the variables are represented by fuzzy numbers whose similarities are compared with the original and modified labels.

Table 1. Modifiers of fuzzy numbers (Juuso, 2016)

\begin{tabular}{llc} 
Fuzzy number & Fuzzy label & Degree of membership \\
\hline$A_{1}$ & extremely & $\mu^{4}$ \\
$A_{2}$ & very $A$ & $\mu^{2}$ \\
$A_{3}$ & $A$ & $\mu$ \\
$A_{4}$ & more or less $A$ & $\mu^{\frac{1}{2}}$ \\
$A_{5}$ & roughly $A$ & $\mu^{\frac{1}{4}}$ \\
\hline
\end{tabular}




\section{Recursive analysis}

All the phases of the data-based LE modelling shown in Figure 1 can be used in the recursive analysis as well. The recursive part focuses on the scaling and the interactions are updated only if needed.

\subsection{Scaling}

The parameter of the scaling functions can be recursively updated by using the norms (1) with the defined orders. The norm values are updated by including new equal sized sub-blocks in calculations since the computation of the norms can be done from the norms obtained for the equal sized sub-blocks, i.e. the norm for several samples can be obtained as the norm of the norms of the individual samples:

$$
\begin{aligned}
\left\|^{K_{s} \tau} M_{j}^{p}\right\|_{p} & =\left\{\frac{1}{K_{s}} \sum_{i=1}^{K_{s}}\left[\left({ }^{\tau} M_{j}^{p}\right)_{i}^{1 / p}\right]^{p}\right\}^{1 / p} \\
& =\left[\frac{1}{K_{s}} \sum_{i=1}^{K_{s}}\left[\left({ }^{\tau} M_{j}^{p}\right)_{i}\right]^{1 / p}\right.
\end{aligned}
$$

where $K_{s}$ is the number of samples $\left\{x_{j}\right\}_{i=1}^{N}$. In automation and data collection systems, the sub-blocks are normally used for arithmetic mean $(p=1)$.

Firstly, the parameters of the scaling functions can be recursively updated with by including new samples in calculations. The number of samples can be increasing or fixed with some forgetting or weighting (Juuso, 2011a).

In the second level, the orders of the norms are redefined if the operating conditions change considerably. The new orders are obtained by using the generalised skewness (2) for the data extended with the data collected from the new situation. If the changes are drastic, the calculations are based on the new data only. The decision of starting the redefinition is fuzzy and the data selection is important.

\subsection{Interactions}

Linear regression and parametric models are used in the recursive tuning of the interaction equations. The set of equation alternatives (Figure 1) is useful in the recursive analysis since the set is validated with domain expertise.

In the first level, the interaction models are not changed. The coefficients are obtained from the data collected from the chosen time period. Uncertainties can be calculated by comparing the coefficients extracted from several short periods.

In the second level, the revised scaling functions may require updates for the interactions as well. However, the re-tuning is started only if the current equations do not operate sufficiently well. The earlier chosen set of alternative equations is used first. New equations are included if new variables become important. The selected variable groups (Figure 1) are analysed first.

Considerable changes in operating conditions mean that the full data-based analysis is needed. This is the third level, which is used to form the model basis for the casebased reasoning (CBR), see (Juuso and Ahola, 2008).

\subsection{Fuzzy logic}

The recursive data analysis produces parameters for the scaling functions and interactions. Uncertainties of the parameters, which are also obtained in the calculations, are used in detecting changes in operating conditions. The detection is based on fuzzy inequalities $<, \leq,=, \geq$ and $>$ between the new fuzzy parameters and the fuzzy parameters of the case. The resulting a 5X5 matrix includes the degrees of membership of these five inequalities for five parameters. The results are interpreted with the natural language interface which provides an important channel in explaining the changes to the users.

\subsection{Smart adaptive systems}

The recursive analysis presented above refines the levels of adaptation. The adaptation to a changing environment has two sub-levels: first updating the scaling functions and then interactions if needed. Similar settings are realised with the set of equation alternatives. The adaptation to a new or unknown application includes the full data-based modelling (Figure 1).

\section{Temporal analysis}

Temporal analysis focused on important variables provides useful information, including trends, fluctuations and anomalies, for decisions on higher level recursive adaptation.

\subsection{Trend indices}

Trend analysis produces useful indirect measurements for the early detection of changes. For any variable $j$, a trend index $I_{j}^{T}(k)$ is calculated from the scaled values $X_{j}$ with a linguistic equation

$$
I_{j}^{T}(k)=\frac{1}{n_{S}+1} \sum_{i=k-n_{S}}^{k} X_{j}(i)-\frac{1}{n_{L}+1} \sum_{i=k-n_{L}}^{k} X_{j}(i),
$$

which is based on the means obtained for a short and a long time period, defined by delays $n_{S}$ and $n_{L}$, respectively. The index value is in the linguistic range $[-2,2]$, representing the strength of both decrease and increase of the variable $x_{j}$. (Juuso, 2011b; Juuso et al., 2009)

An increase is detected if the trend index exceed a threshold $I_{j}^{T}(k)>\varepsilon_{1}^{+}$. Correspondingly, $I_{j}^{T}(k)<-\varepsilon_{1}^{-}$for a decrease (Figure 2). The derivative of the index $I_{j}^{T}(k)$, denoted as $\Delta I_{j}^{T}(k)$, is used for analysing the full set of the triangular episodic representations. Trends are linear if the derivative is close to zero: $-\varepsilon_{2}^{-}<\Delta I_{j}^{T}(k)<-\varepsilon_{2}^{+}$. The concave upward monotonic increase (D) and the concave downward monotonic decrease (B) are dangerous situations, which introduce warnings and alarms. The concave downward monotonic increase (A) and the concave upward monotonic decrease $(\mathrm{C})$ mean that an unfavourable trend is stopping. 


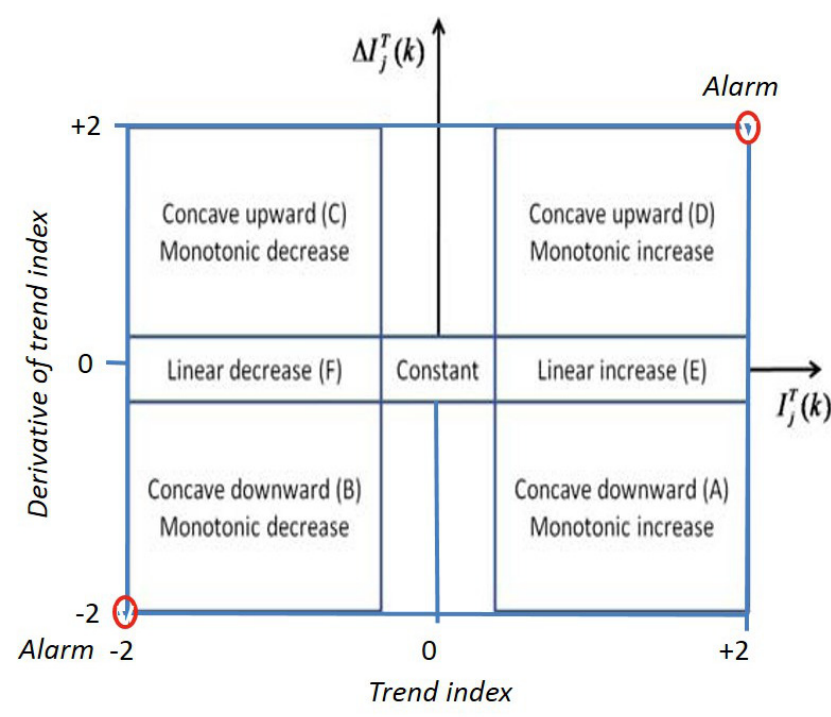

Figure 2. Triangular episodic representations defined by the in$\operatorname{dex} I_{j}^{T}(k)$ and the derivative $\Delta I_{j}^{T}(k)$. index

Severity of the situation can be evaluated by a deviation

$$
I_{j}^{D}(k)=\frac{1}{3}\left(X_{j}(k)+I_{j}^{T}(k)+\Delta I_{j}^{T}(k)\right) .
$$

This index has its highest absolute values, when the difference to the set point is very large and is getting still larger with a fast increasing speed (Juuso et al., 2009). This can be understood as a third dimension in Figure 2.

The trend analysis is tuned to applications by selecting the time periods $n_{L}$ and $n_{S}$. Further fine-tuning can be done by adjusting the weight factors $w_{j}^{T 1}$ and $w_{j}^{T 2}$ used for the indices $I_{j}^{T}(k)$ and $\Delta I_{j}^{T}(k)$. The thresholds $\varepsilon_{1}^{+}=\varepsilon_{1}^{-}=$ $\varepsilon_{2}^{+}=\varepsilon_{2}^{-}=0.5$. The calculations are done with numerical values and the results are represented in natural language.

The trend analysis can be used for the parameters of the scaling functions and interaction coefficients. Trend of the parameters $\alpha_{j}^{-}, \alpha_{j}^{-}, \Delta c_{j}^{-}$and $\Delta c_{j}^{+}$give useful information about changes of the scaling functions. The ranges $[-2,2]$ are $\left[\frac{1}{3}, 3\right],\left[\frac{1}{3}, 3\right] .\left[c_{j}-\min \left(x_{j}\right), \max \left(x_{j}\right)-c_{j}\right]$, respectively.

\subsection{Fluctuations}

The fluctuation indicators, which were introduced to detecting cloudiness and oscillations, are important improvements aimed for practical use. The indicator calculates the difference of the high and the low values of the corrected irradiation as a difference of two moving generalized norms:

$$
\Delta x_{j}^{F}(k)=\left\|{ }^{K_{s} \tau} M_{j}^{p_{h}}\right\|_{p_{h}}-\left\|{ }^{K_{s} \tau} M_{j}^{p_{l}}\right\|_{p_{l}},
$$

where the orders $p_{h} \in \Re$ and $p_{l} \in \Re$ are large positive and negative, respectively. The moments are calculated from the latest $K_{s}+1$ values, and an average of several latest values of $\Delta x_{j}^{F}(k)$ is used as an indicator of fluctuations. (Juuso, 2012b)

\subsection{Changes of operating conditions}

Changes of the scaling functions and interaction coefficients are symptoms of changes in operation. The intelligent trend analysis provides early warning about changes in variable levels, fluctuations and uncertainty. All the variables and intelligent indices are represented in the same range $[-2,2]$, i.e. the same analysis and linguistic interpretation can be applied in all of them. The corresponding levels and their degrees of membership can be used in the fuzzy decision making.

The full analysis is needed fairly seldom although the process changes considerably. For example, new phenomena activate with time in wearing, but the models used in prognostics can be updated by expanding the scaling functions (Figure 3).

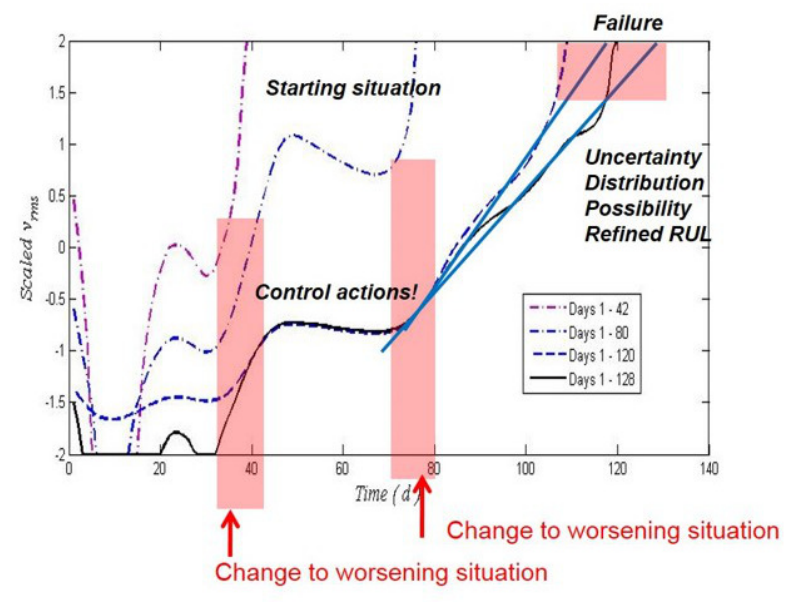

Figure 3. Recursive adaptation in prognostics (Juuso, 2015).

\section{Conclusions}

The nonlinear scaling approach is the main part of the data processing chain which is the integrating part of the natural language interface. The calculations are done in numeric forms, but the levels and all the indices based on them can be represented in natural language. Datadriven extraction of variable meaning and a set of compact models, with not too many variables in individual equations, form the basis for using the recursive data analysis in practical applications. Included uncertainty representations and the natural language interface help in combining the data analysis with the domain expertise. Complexity needs to be reduced and used in a gradually refining way in practical applications. The recursive data analysis also provides more refined steps for the development of smart adaptive systems. 


\section{Acknowledgment}

The author would like to thank the research program Measurement, Monitoring and Environmental Efficiency Assesment (MMEA) funded by the TEKES (the Finnish Funding Agency for Technology and Innovation).

\section{References}

D. Anguita. Smart adaptive systems - state of the art and future directions for research. In Proceedings of Eunite 2001 - European Symposium on Intelligent Technologies, Hybrid Systems and their implementation on Smart Adaptive Systems, July 13-14, 2001, Tenerife, Spain, pages 1-4. Wissenschaftsverlag Mainz, Aachen, 2001.

G. E. P. Box and K. B. Wilson. On the experimental attainment of optimum conditions. Journal of the Royal Statistical Society. Series B, 13(1):1-45, 1951.

J. J. Buckley and T. Feuring. Universal approximators for fuzzy functions. Fuzzy Sets and Systems, 113:411-415, 2000.

J. J. Buckley and Y. Hayashi. Can neural nets be universal approximators for fuzzy functions? Fuzzy Sets and Systems, 101:323-330, 1999.

J. J. Buckley and Y. Qu. On using $\alpha$-cuts to evaluate fuzzy equations. Fuzzy Sets and Systems, 38(3):309-312, 1990.

J. T.-Y. Cheung and G. Stephanopoulos. Representation of process trends - part I. A formal representation framework. Computers \& Chemical Engineering, 14(4/5):495-510, 1990.

M. De Cock and E. E. Kerre. Fuzzy modifiers based on fuzzy relations. Information Sciences, 160(1-4):173-199, 2004.

D. Dubois, H. Prade, and L. Ughetto. Fuzzy logic, control engineering and artificial intelligence. In H. B. Verbruggen, H.J. Zimmermann, and R. Babuska, editors, Fuzzy Algorithms for Control, International Series in Intelligent Technologies, pages 17-57. Kluwer, Boston, 1999.

R. W. Gerlach, B. R. Kowalski, and H. O. A. Wold. Partial least squares modelling with latent variables. Anal. Chim. Acta, 112(4):417-421, 1979.

I. T. Jolliffe. Principal Component Analysis. Springer, New York, 2 edition, 2002. 487 pp.

E. Juuso. Integration of intelligent systems in development of smart adaptive systems: linguistic equation approach. $\mathrm{PhD}$ thesis, University of Oulu, 2013. 258 pp., http://urn.fi/urn:isbn:9789526202891.

E. Juuso and S. Lahdelma. Intelligent scaling of features in fault diagnosis. In 7th International Conference on Condition Monitoring and Machinery Failure Prevention Technologies, CM 2010 - MFPT 2010, 22-24 June 2010, Stratfordupon-Avon, UK, volume 2, pages 1358-1372, 2010. URL WWw. scopus. com.

E. Juuso and S. Lahdelma. Intelligent trend indices and recursive modelling in prognostics. In 8th International Conference on Condition Monitoring and Machinery Failure Prevention Technologies, CM 2011 - MFPT 2011, 20-22 June
2011, Cardiff, UK, volume 1, pages 440-450. BINDT, 2011. www.scopus.com.

E. Juuso, T. Latvala, and I. Laakso. Intelligent analysers and dynamic simulation in a biological water treatment process. In I. Troch and F. Breitenecker, editors, 6th Vienna Conference on Mathematical Modelling - MATHMOD 2009, February 11-13, 2009, Argesim Report no. 35, pages 999-1007. Argesim, 2009. ISBN 978-3-901608-35-3.

E. K. Juuso. Fuzzy control in process industry: The linguistic equation approach. In H. B. Verbruggen, H.-J. Zimmermann, and R. Babuška, editors, Fuzzy Algorithms for Control, International Series in Intelligent Technologies, volume 14 of International Series in Intelligent Technologies, pages 243300. Kluwer, Boston, 1999. doi:10.1007/978-94-011-4405$6 \_10$.

E. K. Juuso. Integration of intelligent systems in development of smart adaptive systems. International Journal of Approximate Reasoning, 35(3):307-337, 2004. doi:10.1016/j.ijar.2003.08.008.

E. K. Juuso. Tuning of large-scale linguistic equation (LE) models with genetic algorithms. In M. Kolehmainen, editor, Revised selected papers of the International Conference on Adaptive and Natural Computing Algorithms - ICANNGA 2009, Kuopio, Finland, Lecture Notes in Computer Science, volume LNCS 5495, pages 161-170. Springer-Verlag, Heidelberg, 2009. doi:10.1007/978-3-642-04921-7_17.

E. K. Juuso. Recursive tuning of intelligent controllers of solar collector fields in changing operating conditions. In S. Bittani, A. Cenedese, and S. Zampieri, editors, Proceedings of the 18th World Congress The International Federation of Automatic Control, Milano (Italy) August 28 - September 2, 2011, pages 12282-12288. IFAC, 2011a. doi:10.3182/20110828-6-IT-1002.03621.

E. K. Juuso. Intelligent trend indices in detecting changes of operating conditions. In 2011 UKSim 13th International Conference on Modelling and Simulation, pages 162-167. IEEE Computer Society, 2011b. doi:10.1109/UKSIM.2011.39.

E. K. Juuso. Integration of knowledge-based information in intelligent condition monitoring. In 9th International Conference on Condition Monitoring and Machinery Failure Prevention Technologies, 12-14 June 2012, London, UK, volume 1, pages 217-228. Curran Associates, NY, USA, 2012a. URL WwW. scopus. com.

E. K. Juuso. Model-based adaptation of intelligent controllers of solar collector fields. In I. Troch and F. Breitenecker, editors, Proceedings of 7th Vienna Symposium on Mathematical Modelling, February 14-17, 2012, Vienna, Austria, Part 1, volume 7, pages 979-984. IFAC, 2012b. doi:10.3182/201202153-AT-3016.00173.

E. K. Juuso. Intelligent methods in modelling and simulation of complex systems. Simulation Notes Europe SNE, 24(1):1-10, 2014. doi:10.11128/sne.24.on.102221.

E. K. Juuso. Recursive data analysis and modelling in prognostics. In 12th International Conference on Condition Monitoring and Machinery Failure Prevention Technologies, CM 
2015 - MFPT 2015, 9-11 June 2015, Oxford, UK, pages 560-567. BINDT, 2015. URL www . proceedings . com. ISBN: 978-1-5108-0712-9.

E. K. Juuso. Informative process monitoring with a natural language interface. In 2016 UKSim-AMSS 18th International Conference on Modelling and Simulation, 6-8 April, 2016, Cambridge, UK, pages 105-110. IEEE Computer Society, 2016. doi:10.1109/UKSim.2016.37.

E. K. Juuso and T. Ahola. Case-based detection of operating conditions in complex nonlinear systems. In M. J. Chung and P. Misra, editors, Proceedings of 17th IFAC World Congress, Seoul, Korea, July 6-11, 2008, volume 17, pages 11142-11147. IFAC, 2008. doi:10.3182/20080706-5-KR1001.01888 .

E. K. Juuso and K. Leiviskä. Adaptive expert systems for metallurgical processes. In S.-L. Jämsä-Jounela and A. J. Niemi, editors, Expert Systems in Mineral and Metal Processing, IFAC Workshop, Espoo, Finland, August 26-28, 1991, IFAC Workshop Series, 1992, Number 2, pages 119-124, Oxford, UK, 1992. Pergamon.

A. Krone and H. Kiendl. Automatic generation of positive and negative rules for two-way fuzzy controllers. In H.-J. Zimmermann, editor, Proceedings of the Second European Congress on Intelligent Technologies and Soft Computing EUFIT'94, Aachen, September 21 - 23, 1994, volume 1, pages 438-447, Aachen, 1994. Augustinus Buchhandlung.

A. Krone and U. Schwane. Generating fuzzy rules from contradictory data of different control strategies and control performances. In Proceedings of the Fuzz-IEEE'96, New Orleans, USA, pages 492-497, 1996.

S. Lahdelma and E. Juuso. Signal processing and feature extraction by using real order derivatives and generalised norms. Part 1: Methodology. The International Journal of Condition Monitoring, 1(2):46-53, 2011. doi:10.1784/204764211798303805.

L. Ljung. System Identification - Theory for the User. Prentice Hall, Upper Saddle River, N.J., 2nd edition, 1999.

J. M. Mendel. Advances in type-2 fuzzy sets and systems. Information Sciences, 177(1):84-110, 2007.

R. E. Moore. Interval Analysis. Prentice Hall, Englewood Cliffs, NJ, 1966.

T. Takagi and M. Sugeno. Fuzzy identification of systems and its applications to modeling and control. IEEE Transactions on Systems, Man, and Cybernetics, 15(1):116-132, 1985.

L. Wasserman. All of Nonparametric Statistics. Springer Texts in Statistics. Springer, Berlin, corr. 3rd edition, 2007.

L. A. Zadeh. Fuzzy sets. Information and Control, 8(June): 338-353, 1965. 